proposed new organization and the National Reference Library for Science and Invention, which is the responsibility of the British Museum, there is no reference to the continued delays in the establishment of this reference library. Neither here nor elsewhere is there any sign in official circles of the real urgency for either the new reference library or a national library policy.

Generally speaking, then, the final report of the Council for Scientific and Industrial Research is purely factual, with few exceptions such as have been noted. It makes little reference to future policy, nor does it comment on a number of the steps which the Government has taken with regard to the organization of civil seience, on which some uneasiness, to say the least, is felt in scientific circles. That uneasiness could easily grow if there is undue delay in dealing with many of the tasks which the Council for Scientific and Industrial Research left uncompleted. What remains to be said is, however, a word of congratulation to the Council and its predecessor, and to those who have worked during the years to contribute to the growing understanding of the important place which science holds in our national life, not only in purely industrial but also in many social and economic matters. The extent to which science has now become a matter for national policy is in great part due to the success of the organization initiated forty years ago, largely as a result of Lord Balfour's foresight and understanding.

\section{EINSTEIN SPACES}

\section{Einstein-Räume}

(Mathematische Lehrbücher und Monographien. Herausgegeben von der Deutschen Akademie der Wissenschaften zu Berlin Institut für Mathematik. 11, Abteilung Mathematische Monographien, Band XVI.) Von A. S. Petrow. Pp. $\mathrm{x}+$ 394. (Berlin: Akademie-Verlag, 1964.) 58 D.M.

T

HIS is a translation into German by Prof. H.-J. Treder of the Russian original, published in 1961. Prof. Petrow has himself revised and enlarged the original for this translation. The translator has increased the bibliography so that it now contains more than 500 references, and has supplied an appendix on the newest developments in the invariant representation of free gravitational fields and the propagation of gravitational waves.

The first chapter deals with tensor analysis in a Riemannian $n$-space; this material is conventional, except for a very detailed discussion of the principal axos of a tensor and an account of Lio groups. In Chapter 2 Einstein's field equations are set down after some historical preliminaries, and an Einstein space defined as one in which the usual vacuum field equations are satisfied. The chapter ends with a useful account of known exact solutions of the vacuum field equations, with or without the cosmological term: here we find the fields of Schwarzschild, Kottler, Weyl, Levi-Civita, Bach, Brinkmann, Kasner, Delsarte, Chou, Narlikar and Karmakar, Einstein and Rosen, Buchdahl, Verma and Roy, and some others.

Chapter 3 contains the matter for which Prof. Petrow is justly famous: Einstein spaces are classified. But here arises a source of petty confusion. It is really the 4 -index Riemann tensor that is being classified, but in general that tensor has 20 independent components and defies classification. The vacuum field equations reduce that number from 20 to 10 , and the classification proceeds. But then the author shows that if we take another related 4-index tensor (actually the Weyl tensor), it has only 10 independent components anyway (irrespective of the field equations), and can be classified in just the same way. In fact, the title of the book is a bit of a misnomer, so far as the essentials of the classification-process are concerned: that process is more powerful than the title seems to imply.

Chapters 4 and 5 are devoted to groups of motions, and the known solutions mentioned above are reviewed in this light. Chapter 6 deals with conformal transformations of Riemannian spaces, and Chapter 7 with the Cauchy (or initial-value problem) in vacuo, for a mass-stream (or dust-cloud) and for a perfect fluid. In Chapter 8 special types of gravitational fields are discussed. These include separable fields ( $\mathrm{d} s^{2}$ splits into a sum of quadratic forms with no co-ordinate occurring in more than one), fields which are separable as the result of conformal transformation, fields with central symmetry and axial symmetry, harmonic fields, cylindrical gravitational fields, and gravitational fields with boundary conditions. Many exercises are distributed throughout the book.

In the days when physicists made crucial experiments with the aid of sealing-wax and string, their physical concepts were merely refined analogues of things in the world about them. Those happy days are gone. Unless one is a mathematician, enjoying mathematics for its own sake, the choice is between a guilty conscience and a headache when it comes to the concepts on which gravitational theory is based. Most people prefer to batten down their consciences and use Newtonian conceptsabsolute space and time, action at a distance with the inverse square law, the laws of motion-all indefensible. The conscientious mind must grapple with a mathematical theory which is impeccable in concept, but so complicated that what little comes out of it may seem scarcely worth the trouble. Some people compromise: working with Einstein's concepts, they try to fit Newtonian language to the results-for example, they see a 'force' in the quadratic term in the equations of a geodesic.

Prof. Petrow does not go in for compromise of that sort. With a clear conscience and no headache, he weaves the mathematical structure pertaining to Einstein's theory, without much apparent concern about physics. Nevertheless, I think he has hit the nail on the head for physicists by insisting that the 4-index Riemann tensor lies at the heart of the matter. This tensor is the gravitational field, just as Maxwell's vectors are an electromagnetic field. For a long time physicists did not like Maxwell's theory, but they have learned to think as he did. Any physicist who wants to think seriously about gravitation must think in terms of the Riemann tensor. Indeed, there is here a good analogy. A Maxwellian field in vacuo may be classified in terms of its two Lorentzinvariants (E.H and $E^{2}-H^{2}$ )-when these both vanish we have the familiar radiation field. Petrow's classification of the Riemann tensor in vacuo (or of the Weyl tensor in general) is, algebraically, a much more erudite affair, but in principle it is similar-it is a question of seeking invariants.

This book is an obvious must for serious students of general relativity. It should be translated into English.

$$
\text { J. L. SYNGE }
$$

\section{PALAEARCTIC GRASSHOPPERS}

\section{Locusts and Grasshoppers of the U.S.S.R. and Adjacent Countries}

By G. Ya, Bei-Bienko and L. L. Mishchenko. Part 1: Pp. $400+x x i$. Part 2: Pp. $291+x x i$. Translated from the Russian. (Jerusalem: Israel Program for Scientific Translations; London: Oldbourne Press, 1963.) 160s. the two volumes.

CINCE its appearance in 1951 the original version of this $\checkmark$ publication has been the standard work on the Acridoidea of the U.S.S.R. The language in which it was 